\title{
Primary extragonadal germ cell tumor: A case report on prostate seminoma
}

\author{
WEI ZHENG, LINA WANG, DEYONG YANG, KUN FANG, XIAOCHI CHEN, XUEJIAN WANG, \\ XIANCHENG LI, ZIYAO LI, XISHUANG SONG and JIANBO WANG
}

Department of Urology, First Affiliated Hospital of Dalian Medical University, Dalian, Liaoning 116011, P.R. China

Received October 7, 2014; Accepted July 7, 2015

DOI: 10.3892/ol.2015.3592

\begin{abstract}
Primary extragonadal germ cell tumors are rare, and $60 \%$ of such cases are seminomas. While the tumors can occur in the mediastinum, thymus, retroperitoneal organs and pineal gland, seminoma originating in the prostate tissue is extremely rare. The present study reports the case of a 54-year-old male with prostate seminoma. The patient was followed up at the First Affiliated Hospital of Dalian Medical University (Dalian, Liaoning, China) from 2001 onwards. The patient received chemotherapy with cyclophosphamide following total resection of the pelvic organs, pelvic lymph node dissection, continent detenial cecum-ascending colic bladder and orchidectomy. The patient experienced considerable post-operative quality of life for $>10$ years and the disease did not reappear. The study indicated that extragonadal seminoma is sensitive to chemotherapy, and that radical surgery and post-operative adjuvant chemotherapy with cyclophosphamide is a reasonable and feasible treatment method.
\end{abstract}

\section{Introduction}

Primary extragonadal germ cell tumors (EGCTs) are rare, accounting for 1-3\% of all GCTs (1), of which $60 \%$ are seminomas. Seminomas are common germ cell tumors (2), with a good prognosis and a mortality rate of $0.1 \%$ (3). The typical clinical feature of seminoma is a progressive increase in testicular size. At present, treatments include surgery, radiotherapy and chemotherapy (4). EGCTs can occur in the mediastinum, thymus, retroperitoneal organs and pineal gland, while seminoma originating in the prostate tissue is extremely rare (5). The clinical symptoms of EGCTs are nonspecific and vary according to tumor location (2). For example, tumors of

Correspondence to: Professor Jianbo Wang, Department of Urology, First Affiliated Hospital of Dalian Medical University, 222 Zhongshan Road, Dalian, Liaoning 116011, P.R. China

E-mail: wangjianbo@dlmedu.edu.cn

Key words: prostate cancer, germ cell tumor, seminoma, cyclophosphamide, chemotherapy the central nervous system, mediastinum and thymus may cause oppression symptoms, tumors of the retroperitoneum usually present as a painless mass (6) and in the prostate, EGCTs may cause progressive difficulty in urination (2). The diagnosis of primary prostate seminoma is difficult based on clinical features alone and thus, pathological examination is the most effective diagnostic technique (5). EGCTs have a good prognosis and a low rate of metastatic potential; even prostate metastasis is uncommon (7).

The present study describes the case of a 54-year-old male with prostate seminoma who presented with symptoms that included progressive difficulty in urination, increased frequency of nocturia and nocturnal incontinence. Ethical approval was obtained from the of First Affiliated Hospital of Dalian Medical University (Dalian, China), and the patient provided informed written consent. The patient was followed up in the First Affiliated Hospital of Dalian Medical University from 2001 onwards.

\section{Case report}

In February 2001, a 54-year-old male patient first presented to the First Affiliated Hospital of Dalian Medical University due to progressive difficulty with urination, increased frequency of nocturia and nocturnal incontinence. Findings from the digital rectal exam indicated an enlarged prostate, with a rougher than normal surface, non-palpable nodules and no central sulcus. The prostate-specific antigen (PSA) level was $3.54 \mathrm{ng} / \mathrm{ml}$ (normal range, 0-4 ng/ml). Ultrasound revealed solid masses within the prostate suggestive of prostate cancer. Computed tomography (CT) scans revealed small sections of low density within the prostate, suggestive of prostate cancer, invading the bladder (Fig. 1). The tumor was $95 \times 72 \times 65 \mathrm{~mm}$ in size. The diagnostic prostate biopsy confirmed the diagnosis of prostate cancer; the tumor cells were arranged in tubular and cribriform structures, and solid nests, with dense nuclear chromatin and one or more large, irregular, distinct nuclei. On February 28, 2001, the patient underwent a total resection of the pelvic organs, pelvic lymph node dissection, continent detenial cecum-ascending colic bladder and orchidectomy. The surgery was successful.

The pathology results revealed that the tumor structure was in line with a diagnosis of seminoma (Fig. 2); the tumor was composed of large neoplastic cells with a centrally located nucleus, containing prominent nucleoli. The nucleus contained 


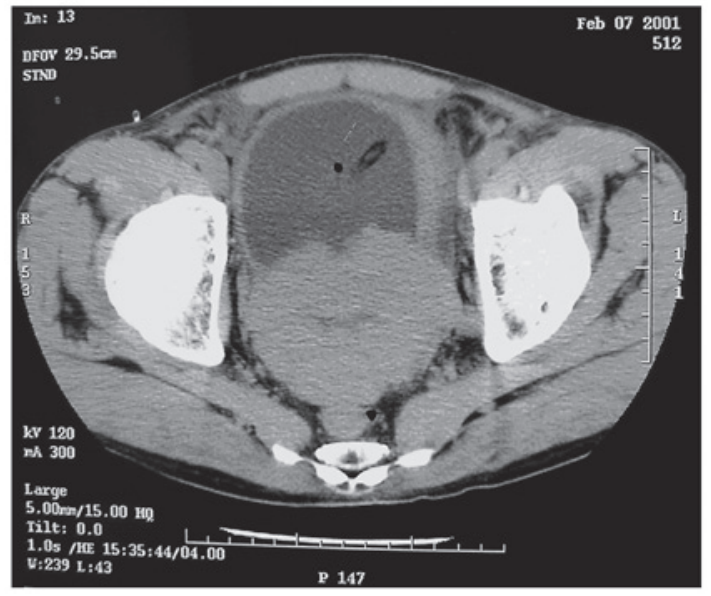

Figure 1. Computed tomography scan showing a large prostate tumor, $9 \mathrm{~cm}$ in diameter.
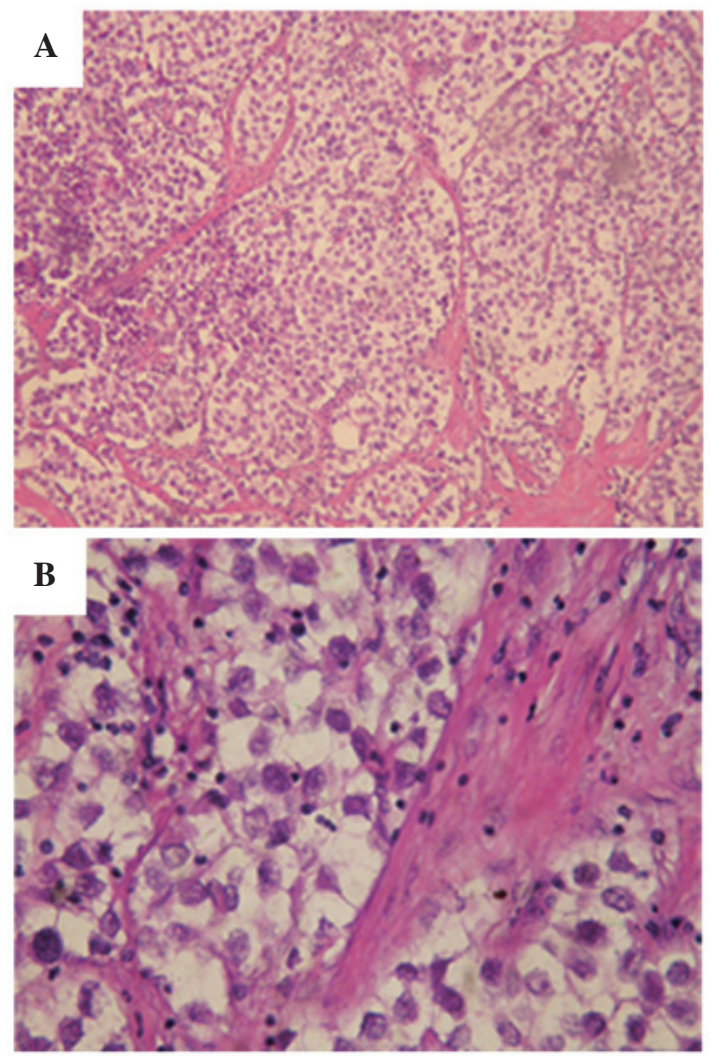

Figure 2. Immunohistochemical analysis of prostate seminoma: The post-operative pathology result. A high number of lymphocytes and inflammatory cell infiltration at the stroma were observed. Hematoxylin and eosin stain; original magnification, (A) x100 and (B) x400.

1-2 nucleoli with rich cytoplasm and stromal lymphocyte infiltration was evident. The tumor involved the wall of the bladder, while the rectal wall and the left ureteral stump were not involved. The lymph nodes of the right and front left iliac vessels and seminal vesicles were involved. Following the removal of the testicles, the pathology report indicated that no tumor cells were present in the testicles, and no evident disorders were identified in the lungs and abdominal organs according to the imaging studies. Therefore, the patient was diagnosed with primary prostate seminoma.

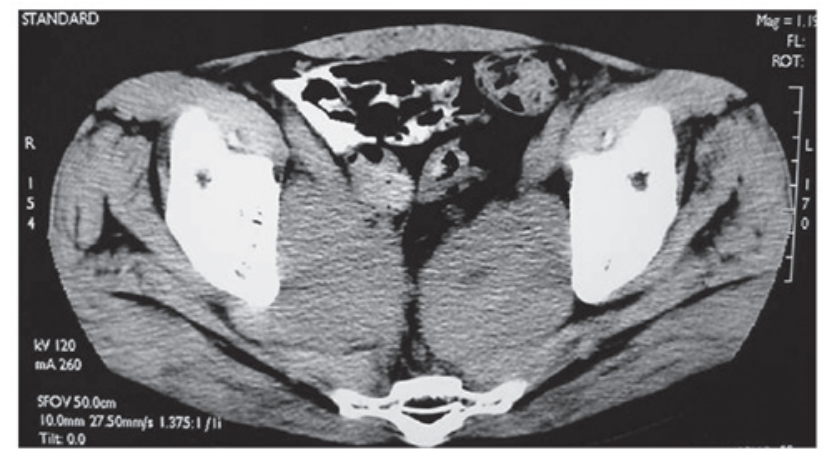

Figure 3. Computed tomography scan showing a pelvic mass suggestive of the metastasis 2 years after the surgery.

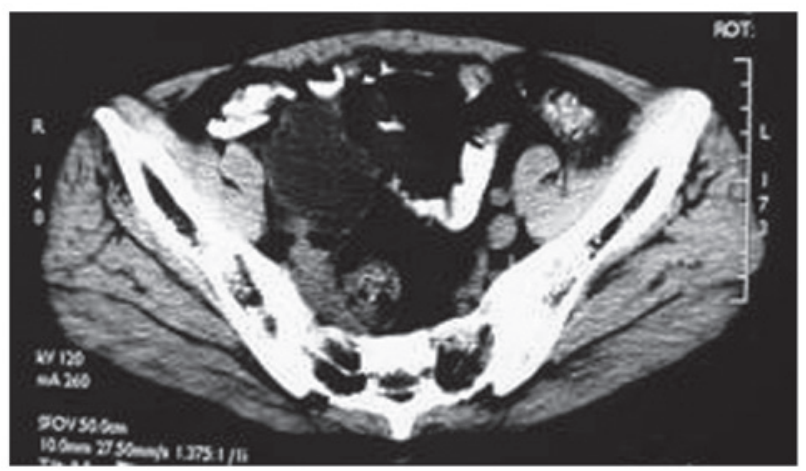

Figure 4. Computed tomography scan showing a decrease in tumor size following cyclophosphamide chemotherapy.

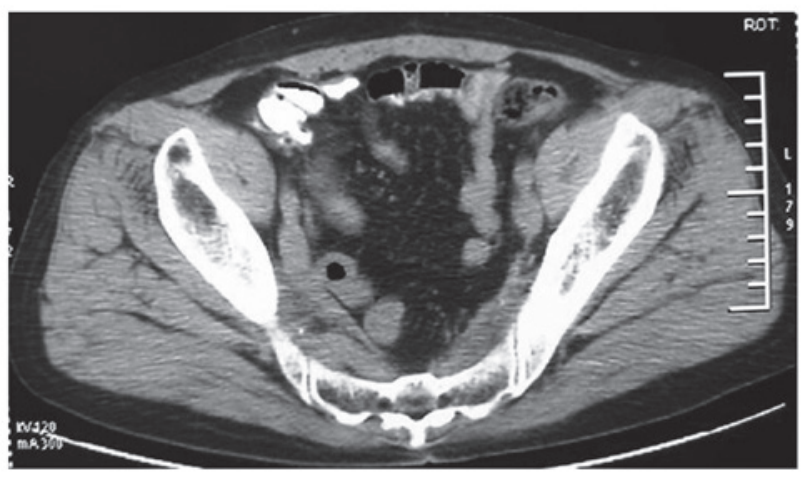

Figure 5. Computed tomography scan showing no recurrence or metastasis of the tumor.

The patient was readmitted to the hospital after 6 months to consolidate the efficacy of intravenous chemotherapy. The initial and consolidation chemotherapy regimens both consisted of 9 cycles of $400 \mathrm{mg}$ cyclophosphamide plus $500 \mathrm{ml}$ normal saline, together with an intravenous glucose tolerance test, once a day, for 1 week. In June 2003, 2 years after the surgery, the pelvic CT scan showed a pelvic mass suggestive of the metastasis (Fig. 3). The lactate dehydrogenase (LDH) tumor marker level (745 IU/1; normal range, 100-300 IU/l) was significantly increased, the total PSA (tPSA) and free PSA (fPSA) levels were normal, and the $\beta$-human chorionic gonadotropin ( $\beta$-HCG; $4.54 \mathrm{ng} / \mathrm{ml}$ ) level was mildly raised. 
Subsequent to the patient receiving cyclophosphamide chemotherapy for 1 week, the symptoms significantly improved and a review of the pelvic CT showed a decrease in tumor size (Fig. 4). The patient then received a total of nine cycles of chemotherapy regimen in line with the previous regimen. In July 2012, 11 years after the surgery, a review of the pelvic CT showed no lumps (Fig. 5), the chest X-ray showed no evident abnormalities, the tests of liver and kidney function, electrolyte levels and coagulation, and the blood routine examination showed no abnormalities, and the associated tumor marker levels $(\beta$-HCG, $\alpha$-fetoprotein, tPSA, fPSA and LDH) were normal. During chemotherapy, the patient occasionally suffered from gastrointestinal symptoms, including nausea, vomiting and anorexia. The patient's condition was improved following symptomatic treatment. Thus far, the follow-up has indicated that the patient eats well and exhibits no other symptoms, such as constipation.

\section{Discussion}

GCTs usually occur in the ovaries and testicles, and account for only $1 \%$ of all malignant tumors, although it is one of the most common malignancies among males aged 30-50 years old. In 2008, 800 new cases of testicular cancer were reported, including 390 mortalities (8). EGCTs account for only 1-3\% of GCTs. The mechanisms generally considered are as follows: i) During embryonic development, primordial germ cells differentiating from the yolk sac endoderm migrate to the urogenital ridge, then reach the gonads, in which course certain cells may remain undifferentiated. ii) During the blastocyst stage of embryonic development, the pluripotent stem cells converting to the germ cells shift to other organs. The vast majority of progenitor cells in these two cases degenerate, but a few non-degenerated cells retain the differentiation potential, and under the stimulation of certain factors they transform into GCTs in the future (9). The former mechanism may explain the cancer incidence in areas such as the mediastinum, thymus, retroperitoneal organs, pineal gland, prostate, seminal vesicles and epididymis, through which the primordial germ cells migrate during the embryonic phase. The latter mechanism may explain the involvement of the liver, stomach, lungs and other unrelated sites (9).

The etiology of primary prostate seminoma is unclear, although there are several possibilities: The origin of the urinary and reproductive systems is similar and the two are closely associated with each other. The urinary and reproductive systems begin to develop between the third and seventh week of development. From the perspective of embryology, the prostate can be defined as a subsidiary organ. Prostate gland cells develop from endoderm cells. One possible inducement of primary prostate seminoma is the translocation of the yolk sac to the gonadal ridge. The prostatic germ cells form tumors. Another hypothesis is that prostatic pluripotent stem cells transform neoplastic cells into gonadal cells, which results in the formation of tumors (10).

Since cases are rare, the standard of care for primary prostate seminoma is controversial. Similar to testicular seminoma, with the exception of surgical treatment, extragonadal seminoma is sensitive to radiotherapy and chemotherapy, which can also confer significant results. Currently, common drugs selected for chemotherapy include cisplatin, etoposide and bleomycin (11).

The patient in the present study was found to exhibit bladder and lymph node metastases, therefore, treatment with radical surgery combined with subsequent chemotherapy was selected. The surgery involved the total resection of the pelvic organs, pelvic lymph node dissection, continent detenial cecum-ascending colic bladder and orchidectomy in order to resect the primary tumor and metastases completely. Continent detenial cecum-ascending colic bladder is a safe and reliable surgical method, with effective urinary diversion, large capacity, internal pressure, high compliance, good urinary control, few complications and a simple procedure. This creates an ideal intestinal neobladder. The patient has experienced considerable quality of life for $>10$ years since the surgery and the disease has not reappeared. Therefore, the selected treatment appears a reasonable option in the present case.

However, there are certain shortcomings, including the results of the post-operative pathology, which are inconsistent with the early prostate biopsy pathology. The present study highlights that findings should be combined with biochemical tests such as that for PSA. PSA in patients with prostate cancer is usually higher than normal (12). If the disease can be recognized and confirmed initially, the correct pathological diagnosis can be obtained. The treatment is then likely to be altered. Chemotherapy is initially used to shrink the tumor. Subsequently, surgical treatment is performed (13). At this time, the range of the surgical resection may be smaller and the post-operative quality of life is improved. This therefore puts a higher demand on the pathological diagnosis.

Testicular seminoma in situ treated with chemotherapy post-operatively is similar. Testicular seminoma is sensitive to radiotherapy and chemotherapy. The simple application of radiotherapy and chemotherapy can also obtain an apparent curative effect. The cure rate is considered to be as high as $80 \%$ when using a good combination chemotherapy with cisplatin $(11,14)$. Although the short-term outcome of combination chemotherapy containing cisplatin is promising, the long-term effect remains unclear $(15,16)$. The prognosis is promising, with a five-year survival rate of $85 \%$, and few recurrences or distant metastases. The lung, liver and brain are the common sites of metastasis, and recurrence and metastasis are sensitive to chemotherapy and radiotherapy $(11,14)$.

Cyclophosphamide was selected as the chemotherapy drug of choice for the present patient. Cyclophosphamide is a common antitumor and immunosuppressive agent. The chemical mechanism mainly has two aspects: The first is the reaction of alkylating groups with the cell functional groups, such as DNA and RNA basic groups, or the reaction with protein functional groups, including mercapto, amino and hydroxyl groups. The alkylating groups take the place of the hydrogen atom leading to mismatch between DNA intrastrand and interstrand cross-linking, chain fractures, termination of replication and loss of protein or physiological activity, resulting in cell apoptosis. The second is the activation and regulation of gene expression changes induced by several signal transduction pathways resulting in interference to cell growth, proliferation and differentiation (17-19).

It has been confirmed that cyclophosphamide can cause spermatogenic impairment. The mechanisms behind this are: 
i) Damage to the genetic material of the germ cells, inducing the apoptosis of spermatogenic cells; ii) interference to the function of A-type spermatogonial cells in self-renewal, proliferation and differentiation; iii) damage to the spermatogenesis microenvironment, including the decrease of seminiferous tubule cell layers, structural disorder, Sertoli cell morphological changes, germ cell sloughing and spermatogenesis disorder; and iv) inhibition of the activity of testis $3 \beta$-hydroxysteroid dehydrogenase (3 $\beta$-HSD) and $17 \beta-\mathrm{HSD}$, thereby causing a reduction of the serum testosterone level and the number of germ cells (20).

Due to the chemical mechanism of cyclophosphamide and its suppression of spermatogenesis, this chemical treatment may be most suitably applied to seminoma patients who have offspring (13). In the present study, cyclophosphamide was used as the chemotherapy drug of choice for $>10$ years. The patient exhibited positive treatment effects, and no relapse or metastasis was identified.

In conclusion, the present study shows a reasonable and feasible treatment method using total pelvic organ resection, pelvic lymph node dissection, continent detenial cecum-ascending colic bladder, testicular resection and post-operative adjuvant chemotherapy treatment with cyclophosphamide for a patient with primary prostate seminoma who already exhibited lymph node metastasis of the surrounding organs. The process of treatment and the $>10$-year follow-up record for this patient provides a valuable reference case and a theoretical basis for the treatment standard of advanced primary prostate seminoma.

\section{References}

1. Bokemeyer C, Nichols CR, Droz JP, Schmoll HJ, Horwich A, Gerl A, Fossa SD, Beyer J, Pont J, Kanz L, et al: Extragonadal germ cell tumors of the mediastinum and retroperitoneum: results from an international analysis. J Clin Oncol 20: 1864-1873, 2002.

2. Krag Jacobsen G, Barlebo H, Olsen J, et al: Testicular germ cell tumours in Denmark 1976-1980. Pathology of 1058 consecutive cases. Acta Radiol Oncol 23: 239-247, 1984.

3. Raghavan D: Testicular cancer: Maintaining the high cure rate. Oncology (Williston Park) 17: 218-228; discussion, 228-229, 2003.

4. Majewski W, Majewski S, Maciejewski A, et al: Adverse effects after radiotherapy for early stage (I,IIa,IIb) seminoma. Radiother Oncol 76: 257-263, 2005.
5. Hashimoto T, Ohori M, Sakamoto N, Matsubayashi J, Izumi M and Tachibana M: Primary seminoma of the prostate. Int J Urol 16: 967-970, 2009.

6. Albany $\mathrm{C}$ and Einhorn LH: Extragonadal germ cell tumors: Clinical presentation and management. Curr Opin Oncol 25: 261-265, 2013.

7. Stein ME, Charas T, Drumea K, Sabo E and Ben-Yosef R: Spermatocytic variant of classic seminoma: A report of five cases and a brief review of the literature. Rambam Maimonides Med J 5: e0021, 2014.

8. Jemal A, Siegel R, Ward E, Hao Y, Xu J, Murray T and Thun MJ: Cancer statistics, 2008. CA Cancer J Clin 58: 71-96, 2008.

9. Peters JA, Beckjord EB, Banda Ryan DR, Carr AG, Vadaparampil ST, Loud JT, Korde L and Greene MH: Testicular cancer and genetics knowledge among familial testicular cancer family members. J Genet Couns 17: 351-364, 2008.

10. Utz DC and Buscemi MF: Extragonadal testicular tumors. J Urol 105: 271-274, 1971.

11. Dueland S, Stenwig AE, Heilo A, Høie J, Ous S and Fosså SD: Treatment and outcome of patients with extragonadal germ cell tumours-the Norwegian Radium Hospital's experience 1979-94. Br J Cancer 77: 329-335, 1998.

12. Wallner LP, Frencher SK, Hsu JW, Chao CR, Nichol MB, Loo RK and Jacobsen SJ: Changes in serum prostate-specific antigen levels and the identification of prostate cancer in a large managed care population. BJU Int 111: 1245-1252, 2013.

13. Alapetite $\mathrm{C}$, Brisse $\mathrm{H}$, Patte $\mathrm{C}$, et al: Pattern of relapse and outcome of non-metastatic germinoma patients treated with chemotherapy and limited field radiation: The SFOP experience. Neuro Oncol 12: 1318-1325, 2010.

14. Gerl A, Clemm C, Lamerz R and Wilmanns W: Cisplatin-based chemotherapy of primary extragonadal germ cell tumors. A single institution experience. Cancer 77: 526-532, 1996.

15. Powles T, Robinson D, Shamash J, Moller H, Tranter N and Oliver T: The long-term risks of adjuvant carboplatin treatment for stage I seminoma of the testis. Ann Oncol 19: 443-447, 2008.

16. Horwich A: Stage I seminoma and carboplatin risks. Ann Oncol 19: 407-408, 2008

17. Waxman DJ: Glutathione S-transferases: role in alkylating agent resistance and possible target for modulation chemotherapy - a review. Cancer Res 50: 6449-6454, 1990.

18. Shulman LN: The biology of alkylating-agent cellular injury. Hematol Oncol Clin North Am 7: 325-335, 1993.

19. Choi YJ, Ok DW, Kwon DN, Chung JI, Kim HC, Yeo SM, Kim T, Seo HG and Kim JH: Murine male germ cell apoptosis induced by busulfan treatment correlates with loss of c-kit-expression in a Fas/FasL- and p53-independent manner. FEBS Lett 575: 41-51, 2004.

20. Schimenti KJ, Hanneman WH and Schimenti JC. Evidence for cyclophosphamide-induced gene conversion and mutation in mouse germ cells. Toxicol Appl Pharmacol 147: 343-350, 1997. 